\title{
Response to Letter from Prof Aspen, re. "Advanced 2D image processing technique to predict hip fracture risk in an older population based on single DXA scans"
}

\author{
F. Jazinizadeh ${ }^{1} \cdot$ J. Adachi ${ }^{2} \cdot$ C. Quenneville ${ }^{1,3}$
}

Received: 14 September 2021 / Accepted: 4 October 2021 / Published online: 19 October 2021

(c) International Osteoporosis Foundation and National Osteoporosis Foundation 2021

\section{Dear Editor,}

Thank you for redirecting the letter regarding the concern for our paper published in Osteoporosis International. It appears that the author has two primary concerns: first, the statement in our paper about the novelty of our approach, and secondly, that their papers were not referenced. We appreciate the opportunity to clarify and address both concerns.

In his letter, Prof. Aspen asserts that we have claimed to be the first to use 2D SSM and SAM. This is incorrect. In the abstract, we state that "a new technique" was presented and assessed, which is true. Our algorithms were new and accounted for a wide variety of factors not included in previous studies. In our introductory section, we presented several previous studies that have used these techniques (references 16-21 in our paper). We stated very clearly that it is a wellestablished and widely used technique. Our literature review did focus on 3D studies, as these have been the more recent area of focus (and builds on the previous body of knowledge from 2D work), whereas Prof. Aspen's previous work in the field was on 2D studies. It appears that he would have preferred us to approach our introduction starting from 2D SAM/SSM, but we believe that this is simply a difference in opinion on the method of writing the background and does not invalidate our introduction or literature review.

This reply refers to the comment available online at https://doi.org/ 10.1007/s00198-021-06096-x.

C. Quenneville

quennev@mcmaster.ca

1 Department of Mechanical Engineering, McMaster University, 1280 Main St West, Hamilton, ON L8S 4L8, Canada

2 Department of Medicine, McMaster University, Hamilton, ON, Canada

3 School of Biomedical Engineering, McMaster University, Hamilton, ON, Canada
We have reviewed the references provided by Prof. Aspden and acknowledge his contributions to this field. He has published on the development of active shape modeling, and we apologize for not including reference to his papers in our literature section. However, upon close inspection, we still believe that our study represents novelty beyond what he has produced in the past (including methods for combining shape and appearance modeling, a unique approach to selection of modes, and enhanced evaluation of the techniques in comparison with clinical metrics).

With our respectful apologies for not including citation of his previous work, we stand by our paper for its value and contributions to the field and believe that any other criticism is the result of differences in writing style and emphasis.

On behalf of all authors,

Dr. Cheryl E. Quenneville

Associate Professor

Department of Mechanical Engineering, School of Biomedical Engineering

McMaster University

Hamilton, ON, Canada

Publisher's note Springer Nature remains neutral with regard to jurisdictional claims in published maps and institutional affiliations. 